INPLASY

PROTOCOL

To cite: Huang et al. Safety and efficacy of robotic versus endoscopic atrial septal defect repair: a systematic review and meta-analysis. Inplasy protocol 202140138. doi:

10.37766/inplasy2021.4.0138

Received: 28 April 2021

Published: 28 April 2021

Corresponding author:

Wang Liang

1191051924@qq.com

Author Affiliation:

1. Inner Mongolia Medical

University, Hohhot 010110, Inner Mongolia; 2.

Department of Cardiology,

Baotou Central Hospital.

Support: There is no source of funds.

Review Stage at time of this submission: Formal screening of search results against eligibility criteria.

Conflicts of interest:

None declared.

\section{Safety and efficacy of robotic versus endoscopic atrial septal defect repair: a systematic review and meta- analysis}

Huang, WM¹; Hou, B2; Li, Q3; Wang, L4.

Review question / Objective: P: Participant:Atrial septal defect. I:Intervention: The Robot surgery for atrial septal defect. C: Comparision: Total endoscopic surgery for atrial septal defect. O: Outcome: The operation related time, thoracic drainage, complications and so on. S: Study design: Revman 5.4 software provided by Cochrane Collaboration Network was used for quality evaluation, bias evaluation and statistical analysis. Objective to screen the randomized controlled studies and case-control studies on the effect of robot versus total endoscopic surgery for atrial septal defect from the establishment of the database to December 2020, and extract the data of operation related time, thoracic drainage, complications, postoperative cosmetic and satisfactory results.

Information sources: We would have to search CJFD, CNKI, VIP, Wanfang Data, PubMed, Cochrane Library clinical trial registration database and EMBASE by computer. The retrieval time was from the establishment of the database to December 2020. Search for academic literature related to topics, not limited to but including journals, treatises, conferences, news reports, articles, and graduation theses. In order to obtain sufficient data, the author of the literature should be contacted if necessary.

INPLASY registration number: This protocol was registered with the International Platform of Registered Systematic Review and Meta-Analysis Protocols (INPLASY) on 28 April 2021 and was last updated on 28 April 2021 (registration number INPLASY202140138).

\section{INTRODUCTION}

Review question / Objective: P: Participant:Atrial septal defect. I:Intervention: The Robot surgery for atrial septal defect. C: Comparision: Total endoscopic surgery for atrial septal defect. O: Outcome: The operation related time, thoracic drainage, complications and so on. S: Study design: Revman 5.4 software 
provided by Cochrane Collaboration Network was used for quality evaluation, bias evaluation and statistical analysis. Objective to screen the randomized controlled studies and case-control studies on the effect of robot versus total endoscopic surgery for atrial septal defect from the establishment of the database to December 2020, and extract the data of operation related time, thoracic drainage, complications, postoperative cosmetic and satisfactory results.

Rationale: In the early 1990s, videoassisted thoracoscopy (VATS) was successfully applied in the field of cardiac surgery, and then encountered some problems, such as insufficient field of vision, poor accuracy, fatigue and difficulty in operation. On this basis, Da Vinci robotic surgery system was introduced into China. Compared with other surgical fields, robotic minimally invasive cardiac surgery is a disruptive technological innovation, but its technology and related equipment are still improving and developing. At present, robot surgery compared with total endoscopy is controversial in clinical practice. For example, the learning curve of robot surgery technology system is long, lack of a lot of clinical experience, high cost, and the effect needs systematic evaluation.

Condition being studied: The effect of robot versus total endoscopic surgery for atrial septal defect.

\section{METHODS}

Search strategy: We searched CJFD, CNKI, VIP, Wanfang Data, PubMed, Cochrane Library clinical trial registration database and EMBASE by computer. The retrieval time was from the establishment of the database to December 2020. We used subject words (expanded) combined with free words, The Chinese and English subject terms are determined by the MeSH thesaurus provided by sinomed and PubMed, and the free words are determined by the keywords used in the previous literature. Key words in Chinese include: atrial septal defect, video assisted thoracoscopy, robotics, robotic surgery, cardiac surgery, minimally invasive surgery, minimally invasive surgery. The key words in English were ASD, atomic partial defect, endoscopes, thoracoscopes, thoracoscope, robot assisted, robotic surgical procedures and minimally invasive surgical procedures. Objective to collect the comparative analysis of the clinical effect of robotic and endoscopic atrial septal defect surgery. Taking PubMed as an example, the retrieval strategy is as follows: "Atrial Septal Defect" [Mesh]OR"persistent ostium primum" [Mesh]OR "Heart Septal Defects, Atrial" [Mesh]OR"persistent ostium primum" [tw] OR" atrial septal defect")AND("Robotic Surgical Procedures"[Mesh]OR"Robot-Assisted Surgery"[Mesh]OR "Robotics"[Mesh]OR" Thoracoscopes"[Mesh]OR"Pleuroscopes" [Mesh])OR"Endoscopes"[Mesh]).

Participant or population: The robot group and endoscopic group included in this study come from different countries and regions and are divided into different age groups. There was no significant difference in general information (including age, gender, height and body surface area) between the two groups. Investigation of domestic research found that all the patients communicated with each other before operation, obtained their own consent and signed informed consent.

Intervention: The Robot surgery for atrial septal defect.

Comparator: Total endoscopic surgery for atrial septal defect.

Study designs to be included: The randomized controlled studies and casecontrol studies on the effect of robot versus total endoscopic surgery for atrial septal defect should be included.

Eligibility criteria: The standard of robot group only includes the patients who only use robotic surgery system to repair atrial septal defect, while the standard of total endoscopy group only includes the patients 
who only use endoscopic surgery system to repair atrial septal defect.

Information sources: We would have to search CJFD, CNKI, VIP, Wanfang Data, PubMed, Cochrane Library clinical trial registration database and EMBASE by computer. The retrieval time was from the establishment of the database to December 2020. Search for academic literature related to topics, not limited to but including journals, treatises, conferences, news reports, articles, and graduation theses. In order to obtain sufficient data, the author of the literature should be contacted if necessary.

Main outcome(s): Systemic circulation time; thoracic drainage, complication; Postoperative Residual Shunt; postoperative hospital stay; R-VAS score; cosmetic effect.

Additional outcome(s): Patient satisfaction; survival time.

Data management: Through three ways (manual import, online search import, search result generation file import) to import references, and then we search, duplicate and edit the local literature database. First of all, a separate group is established to store the references of the article. Secondly, after opening Noteexpress, drag the reference to the window of Noteexpress, the document will be automatically updated by the software, and the article name, author, issue number and volume number will appear. If it cannot be updated automatically, you can choose to update the file manually. Finally, after setting the output format, open the word where you want to insert the reference. In the top line, there will be a Noteexpress button, and then click the insert citation button. At this time, select the document you want to insert in Noteexpress, go to word, click the position where you want to insert the reference, and then click Insert citation to insert the reference, And the bibliography of references will be automatically generated at the end of the article.
Quality assessment / Risk of bias analysis: Two researchers independently searched and strictly screened the literatures according to the inclusion and exclusion criteria. When there were differences, We could discuss and solve them, and solicit the opinions of other researchers when necessary. The methodological quality of randomized controlled trials was evaluated according to the Cochrane Collaboration Network System reviewer's manual. Revman 5.4 software provided by Cochrane Collaboration Network was used for statistical analysis.

Strategy of data synthesis: The odds ratio or and $95 \% \mathrm{Cl}$ were calculated for count data, and MD and $95 \% \mathrm{Cl}$ were used for measurement data. If there is no statistical heterogeneity between the studies, $\mathbf{P} \geq \mathbf{0 . 1 0}$ or I $2<50 \%$, the fixed effect model was used for meta-analysis. The random effect model was used for analysis when the heterogeneity was significant $(P \leq 0.10$ or 12>50\%). When the heterogeneity is too large, descriptive analysis is used.

Subgroup analysis: We will take the indicators (postoperative hospital stay, incidence of postoperative complications, intervention rate of postoperative complications, incidence of postoperative pneumothorax, incidence of postoperative subcutaneous emphysema, incidence of postoperative pleural effusion) of thoracoscopic robotic surgery group and total endoscopic surgery group as subgroup analysis.

Sensitivity analysis: We found that the following outcomes were heterogeneous (I $2>50 \%, P<0.1$ ): cardiopulmonary bypass time, aortic occlusion time, ventilator assisted ventilation time, postoperative drainage volume, incision satisfaction. Sensitivity analysis was carried out to find the source, and it was found that the heterogeneity came from two literatures (). After eliminating these two literatures, the heterogeneity decreased significantly, but still existed. Therefore, the fixed effect model was used according to the standard, and the results were combined. Finally, we 
believe that the lack of included studies is an important reason for the heterogeneity.

Language: All studies involved in this metaanalysis are limited to Chinese and English.

Country(ies) involved: China, USA, Canada, Turkey.

Other relevant information: Wang Liang's resume: while completing many basic experiments and clinical applications in the field of cardiovascular surgery, he has completed more than 10 scientific research projects, including 3 scientific and technological progress awards of Baotou City and 1 scientific and technological progress award of Inner Mongolia Medical Association. He has published more than 20 papers in various domestic public journals, including more than $\mathbf{1 0}$ articles in the professional core journals. In March 2017, he cooperated with the cardiovascular surgery department of the General Hospital of the Chinese people's Liberation Army and was awarded the "cardiovascular surgery academician workstation" by the regional government and Baotou municipal government. He has been to Germany, the United States, Canada and other places for many times to participate in international academic conferences and research exchanges of cardiovascular disease.

Keywords: ASD, Atrial Septal Defect, End os copes, Thoracoscopes, Thoracoscopy, Robot-assisted, Robotic Surgical Procedures, Minimally Invasive Surgecal Procedures.

Contributions of each author:

Author 1 - Huang Weimin - The author drafted the manuscript.

Email: 1191051924@qq.com

Author 2 - Hou Biao - The author provided statistical expertise.

Author 3 - Li Qin - The author contributed to the development of the selection criteria, and the risk of bias assessment strategy.

Author 4 - Wang Liang - The author read, provided feedback and approved the final manuscript. 